\section{Estamos realmente detectando violência familiar contra a criança em serviços de saúde? A experiência de um serviço público do Rio de Janeiro, Brasil}

\author{
Are we really detecting violence in families \\ of children visiting our health services? \\ The experience of a public health service \\ in Rio de Janeiro, Brazil
}

Anna Tereza M. Soares de Moura 1 Michael E. Reichenheim 1

\section{Introdução}

Domestic violence, particularly in childhood, is a growing public health concern. Information on morbidity is mostly underreported due to constraints in case detection. This paper analyzes the frequency of events measured actively by outpatient services as compared to spontaneous reports. Information on violence was assessed for 245 families from April to June 2001 using the Conflict Tactics Scales: Parent-Child Version (CTSPC) and the Revised Conflict Tactics Scales (CTS2). Cases referred to the Social Work Department provided the caseload for the active search period (12 months). There was a high prevalence of physical violence in the couple, with serious events occurring in $17.0 \%$ of the families. In relation to children, cases of " $m i$ nor" physical aggression were reported in $46.0 \%$ of families and serious cases in 9.9\%. The spontaneously identified prevalence was 3.3\%. This case study demonstrates the missed opportunities for detection and calls attention to the need to review the approach to domestic violence by health services.

Child; Domestic Violence; Health Service
O perfil das causas de mortalidade no Brasil vem se modificando nas últimas décadas e, à semelhança de países desenvolvidos, as principais causas de óbito atualmente são as doenças cardiovasculares, neoplasias e causas externas (Ministério da Saúde. Mortalidade Proporcional por Grupo de Causas. Brasília; 2000). Estas últimas, no entanto, vêm apresentando uma tendência crescente nos últimos anos, com um aumento expressivo no coeficiente de mortalidade por causas externas na faixa etária de 0 a 19 anos 1,2,3. Segundo o Ministério da Saúde (MS), as causas externas foram responsáveis por aproximadamente 120 mil mortes em 2001, representando cerca de 15,0\% dos óbitos registrados no país (Ministério da Saúde. Política Nacional de Redução da Morbimortalidade por Acidentes e Violências. Brasília; 2001).

A dinâmica do perfil sanitário brasileiro também tem implicado uma mudança radical nos grupos etários atingidos. As mortes por causas externas são emblemáticas, pois atingem preferencialmente os mais jovens 4,5 . No Rio de Janeiro, por exemplo, já no início da década passada, 30,0\% dos anos potenciais de vida perdidos se deviam às causas externas 6 . Dados oficiais mais recentes continuam confirmando este cenário. No ano de 1999, um quinto do total de óbitos de crianças e jovens brasileiros até 19 anos foi por causas externas, mar- 
cadamente a primeira causa mortis nesta faixa etária 7 . Vale ressaltar que, no contexto das causas externas, expressiva parte dos óbitos se deve às violências. Estudos acrescentam ainda que as crianças são as vítimas preferenciais da violência que ocorre dentro de casa, enquanto a extradomiciliar atinge preferencialmente os adolescentes e adultos jovens (Ministério da Saúde. Política Nacional de Redução da Morbimortalidade por Acidentes e Violências. Brasília; 2001).

Dimensionar a magnitude da violência familiar ainda é um desafio para os pesquisadores da área, sendo difícil estimar sua freqüência de ocorrência 8,9 . No entanto, há evidências crescentes de que o problema é significativo e universal 9,10,11,12,13,14,15,16. Nos Estados Unidos, por exemplo, cerca de 1,5 milhão de crianças são vítimas de maus-tratos e $6,3 \%$ dos casais vivenciam alguma forma de violência grave a cada ano ${ }^{8}$. No Brasil, estudo recente mostra que a incidência de agressões físicas graves contra mulheres grávidas pode chegar a 16,5\% 13 .

Nos últimos anos tem havido um crescente reconhecimento de que os serviços de saúde têm um importante papel no enfrentamento da violência intrafamiliar 17. O setor saúde vem sendo chamado a responder e participar do enfrentamento desta questão, sendo diversas as suas possibilidades de atuação 18,19. Através de um olhar cada vez mais atento dos profissionais da área, vislumbra-se a importância da elaboração de propostas de ação referentes à prevenção, detecção e ao acompanhamento do número crescente de vítimas da violência familiar 20 . Observa-se também uma maior motivação em criar no setor estruturas adequadas à condução dos casos detectados, respeitando-se as características de cada serviço ou localidade por meio do conhecimento do perfil e necessidades de sua clientela 21 . Os serviços de saúde podem desempenhar um papel importante na implementação das modificações necessárias por se apresentarem como locais propícios à revelação dos casos de violência familiar. Esta prerrogativa pode contribuir de maneira decisiva, não só na identificação dos eventos que merecem atuação imediata ou mediata, mas também para um conhecimento mais consistente da magnitude do problema. A revelação de casuísticas fidedignas é essencial e se apresenta como uma etapa inicial para o desenvolvimento de programas de atuação 22,23,24. A elaboração de processos sistemáticos de vigilância dentro dos serviços, acoplada ao desenvolvimento de ações em rede integrando os vá- rios setores envolvidos, seriam fundamentais para a abordagem plena da violência familiar. Estudos revelam que os dados gerados no âmbito da saúde tendem a ser mais completos do que os provenientes de outros setores, tais como os oriundos dos setores policial e jurídico 19.

A violência familiar já vem sendo enfrentada há algumas décadas em países desenvolvidos e algumas estratégias para identificação de casos em serviços de saúde foram sugeridas e aplicadas. Estudos mostram que a inclusão de perguntas sobre eventos violentos nas fichas de atendimento de emergência pode chamar a atenção dos profissionais quanto à necessidade de abordar esta questão, aumentando a possibilidade de detecção 25. Benger \& Pearce 26 mostraram que a existência de um fluxograma anexado ao boletim de emergência para avaliar a presença de lesões intencionais em crianças abaixo de 5 anos com história de traumatismo elevou de forma significativa as suspeitas dos profissionais do setor. Em um estudo realizado em clínicas de pré-natal, Wiist \& McFarlane 27 mostraram que, após o desenvolvimento e a aplicação de um protocolo direcionado para violência familiar, a detecção de casos aumentou cerca de oito vezes.

Apesar do crescente reconhecimento da violência no âmbito familiar como um problema de saúde pública, manifestado abertamente por meio de explícitas convocações para que sejam debatidas e desenvolvidas políticas macroestruturais 18, a situação ideal ainda está longe de ser alcançada. Os profissionais da área da saúde tendem a subestimar a importância da violência familiar 28 , sendo ainda muito precária a detecção de casos em serviços de atenção primária, principalmente quando se leva em conta a elevada freqüência do evento. Vários autores apontam que poucos esforços têm sido empregados para modificar este cenário 24,29.

Enfocando o cenário brasileiro, é necessário que se indague a respeito da situação atual, especialmente quando se considera uma história bem mais recente de estudos nesta área. A proposta de contrastar os resultados provenientes de uma busca ativa com a casuística espontânea de violência familiar contra a criança, se justifica pela possibilidade de levantar um debate sobre o processo de detecção. Desta forma, se colocaria em pauta toda a questão das oportunidades perdidas de detecção e do subdimensionamento da violência familiar. É nesta perspectiva que se insere o presente estudo de caso que, se por um lado, está calcado em um 
serviço de saúde ambulatorial específico, de outro, pode ser ilustrativo ao revelar uma situação que pode ser bem mais comum do que o imaginado. Este ponto é retomado na seção de Discussão à luz dos resultados encontrados.

\section{Métodos}

\section{Cenário do estudo}

O estudo foi realizado no ambulatório de pediatria do Hospital Universitário Pedro Ernesto que é vinculado à Faculdade de Medicina da Universidade do Estado do Rio de Janeiro. Este serviço presta assistência a crianças de 1 a 12 anos, moradoras de áreas adjacentes ao hospital e também referidas de outras localidades da cidade. O ambulatório possui equipe multiprofissional, oferecendo atendimento em pediatria geral e diversas especialidades. Também são desenvolvidas atividades de ensino no serviço para alunos de graduação e pós-graduação em pediatria e áreas correlatas. O atendimento das crianças agendadas para consultas de puericultura e pediatria geral é realizado pelos alunos do último ano do internato rotatório da Faculdade de Medicina e pelos residentes de pediatria de primeiro e segundo anos, com supervisão dos médicos e professores do setor.

\section{Busca ativa de casos de violência} familiar contra a criança

Para a busca ativa da presença de violência familiar contra a criança foi realizado um estudo representativo da clientela de primeira vez do ambulatório de pediatria, entre abril e junho de 2001. A cada dia realizou-se uma amostragem aleatória dos prontuários fornecidos para consultas, considerando-se somente as crianças cujos acompanhantes possuíam vínculos de cuidado com as mesmas. A amostra se restringiu à demanda espontânea ao setor de pediatria geral, sendo excluídas as crianças que traziam como queixa principal sinais ou sintomas que sugerissem patologias prévias, ou aquelas que procuravam o ambulatório com encaminhamento direcionado à avaliação da presença de algum agravo crônico. A amostra representou aproximadamente $70,0 \%$ das crianças que procuraram o ambulatório de pediatria para primeira consulta no período de coleta de dados. As mães representaram a grande maioria dos respondentes $(88,5 \%)$. O tamanho amostral final foi de 245 questionários aplicados, não tendo ocorrido qualquer recusa à participação.
Um questionário estruturado foi aplicado sob forma de entrevistas face a face em local previamente reservado, mediante o aceite do responsável e assinatura de um termo de consentimento. As entrevistas duravam aproximadamente trinta minutos e eram realizadas antes da consulta agendada, visando a evitar perdas das famílias selecionadas para compor a amostra. Sempre que possível, as crianças quer aquela que originava o atendimento (aqui denominada criança-índice), quer algum irmão também presente - realizavam atividades lúdicas em salas separadas durante a entrevista.

Para avaliar a violência familiar vivenciada pela criança-índice foram consideradas duas relações do contexto familiar, a saber: aquela ocorrendo entre os pais ou responsáveis, e destes para com a própria. Na aferição dos eventos direcionados à criança foi utilizado o instrumento Conflict Tactic Scales: Parent-Child Version (CTSPC) 30. Este é composto de um módulo principal cujas escalas visam a captar algumas dimensões da violência familiar existentes na relação entre pais e filhos, a saber, formas não violentas de disciplina (4 itens englobando alguns atos: colocar de castigo, dialogar ou retirar regalias), agressão psicológica (5 itens: xingar, gritar ou ameaçar) e violência física (13 itens contemplando atitudes como dar tapas ou beliscões, sacudir, chutar, queimar, bater com a mão ou com objetos duros). Respeitando um crescendo na gravidade dos atos perpetrados, a última escala é ainda subdividida em três, separando os atos considerados como de punição corporal (6 itens), maus-tratos físicos menores (3 itens) e maus-tratos físicos graves (4 itens). Vale apontar que neste estudo não foi utilizado o módulo suplementar da CTSPC que aborda ainda as táticas utilizadas para disciplinamento infantil na semana anterior à entrevista, situações de negligência e abuso sexual. Para estimar a presença de violência familiar no casal utilizou-se a Revised Conflict Tactic Scales (CTS2) 31. O instrumento é composto por quatro escalas cobrindo atos de agressão psicológica (8 itens: xingar, ignorar, gritar, ofender ou ameaçar), violência física (12 itens: dar um murro, tapa ou uma surra, estrangular, chutar, empurrar ou queimar), coerção sexual (7 itens onde o agressor pode insistir, ameaçar ou forçar o ato sexual) e lesão corporal (6 itens onde se questiona a presença de manchas roxas, dores no corpo ou fraturas). Os itens que compõem estas escalas são também subdivididos em menores e graves, de acordo com sua seriedade. Os dois instrumentos foram previamente adaptados para uso no Brasil 32,33,34. 
A CTS2 foi aplicada exclusivamente quando havia relato de um vínculo marital estável nos 12 meses anteriores à entrevista, mesmo na ausência de laços de parentesco do companheiro com a criança-índice. Vale ressaltar que uma replicação foi realizada em 22 questionários visando a avaliação da qualidade da informação. As estimações de concordância entre os observadores nas diversas dimensões referentes à CTS2 calculadas por intermédio do kappa mostraram-se todas acima de 0,7 , o que pode ser considerado de moderado a bom segundo os critérios propostos por Shrout 35 . Em relação às escalas da CTSPC, o nível de concordância foi razoável, com kappas variando entre 0,4 e 0,6.

Os questionários foram codificados e digitados utilizando-se o programa Epi Info versão 6.04. O processamento e a análise dos dados foram realizados em Stata 7.0

\section{Casuística espontânea encaminhada ao serviço social do ambulatório de pediatria}

A casuística do serviço social se constitui primordialmente de casos identificados e referidos pelos vários profissionais do ambulatório de pediatria. Todos os casos suspeitos de violência familiar contra a criança são encaminhados ao setor. O serviço social tem, entre suas atribuições, a consolidação e o registro dos casos segundo a classificação proposta pelo MS (Política Nacional de Redução da Morbimortalidade por Acidentes e Violências. Brasília; 2001), que contempla a agressão física, o abuso sexual, a agressão psicológica e a negligência como formas de maus-tratos contra a criança.

A busca de registros de casos de violência familiar contra a criança no serviço social se restringiu à mesma janela temporal da busca ativa. Assim, os casos espontâneos considerados no estudo foram os encaminhados e registrados no setor nos doze meses que antecederam a coleta de dados, tomando-se o mês de maio como marco por ser central ao período de recordatório coberto pelas CTS (Conflict Tactis Scales).

\section{Questões éticas}

O protocolo de pesquisa foi submetido ao Comitê de Ética do Hospital Universitário Pedro Ernesto para sua aprovação prévia. As famílias selecionadas para participação na pesquisa eram informadas acerca dos objetivos da mesma, do caráter confidencial das informações obtidas e, só então, convidadas a assinar o termo de consentimento. No caso de detecção de casos de violência familiar durante a aplicação dos questionários, ou quando as famílias mos- travam situação de conflito evidente, era oferecida a possibilidade de uma entrevista especializada e encaminhamento ao serviço social do ambulatório de pediatria.

\section{Resultados}

A Figura 1 mostra a magnitude dos vários matizes de violência familiar contra a criança quando perpetrada pela mãe, pelo pai ou companheiro, detectada durante o processo de busca ativa. Observa-se que a prevalência de período (12 meses) dos atos cometidos pela mãe foi sempre superior à do pai (companheiro) e que existe um padrão descendente à proporção que os atos perpetrados vão se tornando mais graves.

A disciplina não violenta como forma de resolução de situações de desobediência da criança foi reportada pela maioria das entrevistadas (98,9\%; IC95\%: 96,7-99,9). A prevalência da agressão psicológica quando perpetrada pela mãe também alcançou altos níveis $(94,2 \%$; IC95\%: 91,3-97,2). Chama a atenção que, na relação entre a mãe e a criança, o uso de castigo corporal e de agressão psicológica foi reportado por mais de $90,0 \%$ das respondentes, indicando a co-ocorrência destes comportamentos. Os itens de maior prevalência nestas dimensões foram, respectivamente, "dar uma palmada no bumbum" e "falar alto ou berrar". Em relação ao mau trato físico menor, $24,7 \%$ das mães (IC95\%: 19,2-30,1) referiram ter batido "com chinelo ou outro objeto duro em região diferente do bumbum", pelo menos uma vez em seus filhos. O uso de atos violentos graves ocorreu em 9,9\% (IC95\%: 6,1-13,6) na relação da mãe com a criança-índice e em 7,9\% (IC95\%: $4,2-11,7)$ na do pai com a criança-índice. Quando questionadas a respeito de um dos itens da escala, 4,5\% (IC95\%: 1,9-7,2) das respondentes relataram "ter batido muito, sem parar, o máximo que conseguiram" pelo menos uma vez e 3,7\% (IC95\%: 1,3-6,1) disseram tê-lo feito em mais de uma ocasião.

O perfil dos conflitos existentes entre o casal é apresentado na Figura 2. Observa-se que as prevalências de período (12 meses) nesta relação também foram decrescentes no sentido dos atos menores para os mais graves. As mulheres apresentaram prevalências superiores de agressão psicológica quando comparadas aos homens, tanto na subescala menor quanto na grave. Este quadro se inverte nas dimensões de coerção sexual e injúria corporal, com os companheiros sendo responsáveis por um número maior de atos perpetrados. Em relação à 
Figura 1

Prevalência de violência contra a criança quando perpetrada pelos pais, revelada por meio da busca ativa com o uso da Conflict Tactics Scales: Parent-Child Version (CTSPC), no período de abril a junho de 2001, ambulatório de pediatria do Hospital Universitário Pedro Ernesto, Rio de Janeiro, Brasil.

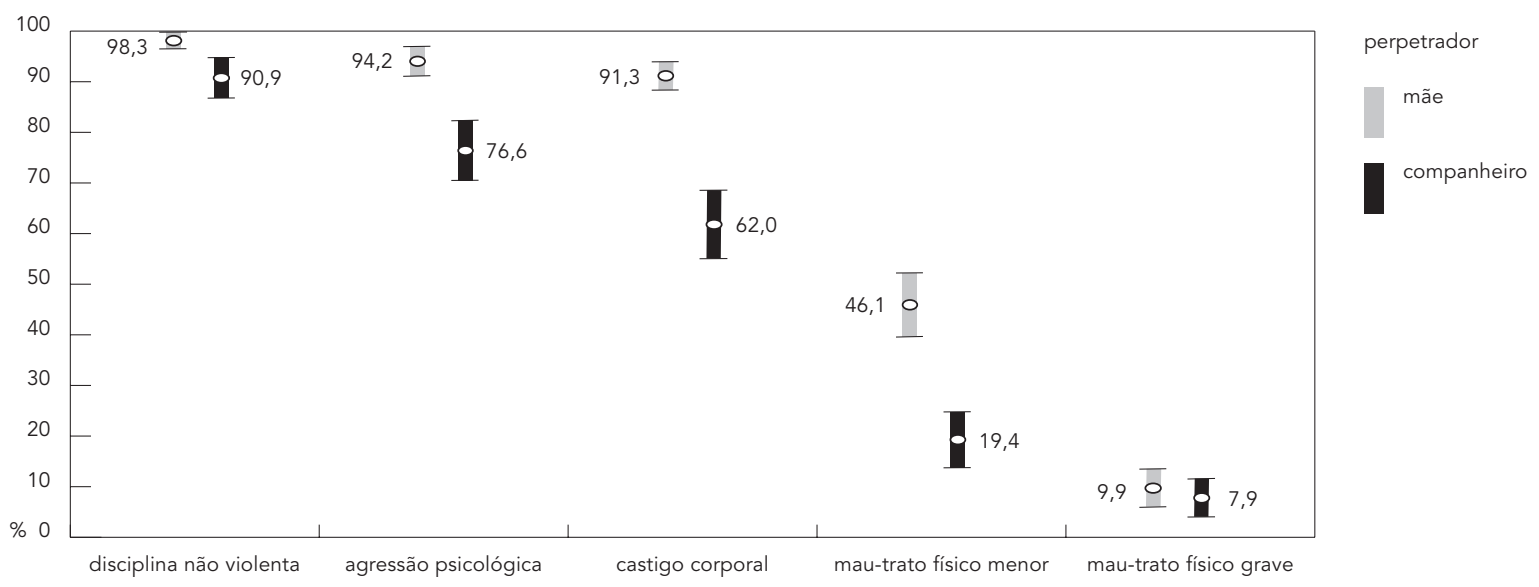

Nota: as barras indicam intervalos de confiança de $95 \%$.

Figura 2

Prevalência de violência entre o casal, revelada por meio da busca ativa com o uso da Revised Conflict

Tactics Scales (CTS2), no período de abril a junho de 2001, ambulatório de pediatria do Hospital Universitário

Pedro Ernesto, Rio de Janeiro, Brasil.

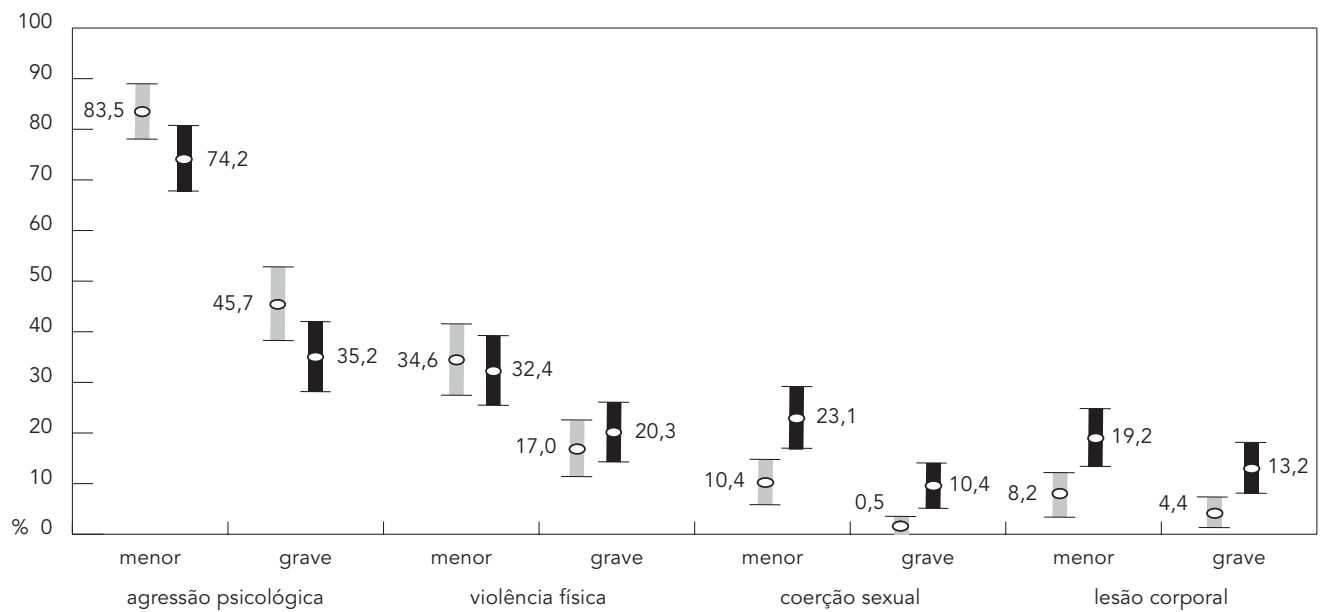

mulher $\rightarrow$ homem

homem $\rightarrow$ mulher

agressão psicológica

violência física

coerção sexual

lesão corporal

Nota: as barras indicam intervalos de confiança de $95 \%$. 
agressão física menor, existe uma superposição entre a mulher praticando a violência (34,6\%; IC95\%: 27,6-41,6) e o homem $(32,4 \%$; IC95\%: 25,5-39,3).

O emprego de atos violentos considerados graves entre o casal foi expressivo, a saber, $17,0 \%$ (IC95\%: 11,5-22,5) para as mulheres perpetrando e 20,3\% (IC95\%: 14,4-26,3) para os homens. Alguns itens que compõem esta subescala merecem um detalhamento. A prevalência do item referente ao "uso de arma de fogo ou faca" durante os conflitos maritais foi de 5,0\% (IC95\%: 1,9-7,4) e $8,0 \%$ (IC95\%: 4,2-11,7) quando a mulher e o homem eram respectivamente, os agressores. O relato de "murros" na resolução de conflitos foi de 2,7\% (IC95\%: 0,3-5,1) quando ocorridos pelo menos uma vez, chegando a $7,1 \%$ (IC95\%: 3,3-10,9) em mais de uma ocasião quando o companheiro era o agressor. O item referente ao uso de "chutes" alcançou, respectivamente, 1,6\% (IC95\%: 0,2-3,5) e 7,7\% (IC95\%: 3,8-11,6) quando a mulher e o homem eram os agressores.

A Tabela 1 mostra a distribuição dos casos registrados e encaminhados ao serviço social, de junho de 2000 a maio de 2001. Foram identificados 41 casos de violência familiar contra a criança, o que projeta uma prevalência de 3,3\% (IC95\%: 2,4-4,5). Esta casuística é composta principalmente de casos graves e exuberantes na sua apresentação, capazes de despertar a necessidade de encaminhamento por parte do profissional de saúde. Ressalta-se a magnitude das agressões físicas e abuso sexual identificados e referidos durante os 12 meses equivalentes ao período coberto pela busca ativa. Vale salientar que os casos se referem apenas à violência direcionada à criança-índice, não tendo sido encaminhado ao serviço social sequer uma situação de violência existente entre outros componentes do núcleo familiar.

\section{Discussão}

Claramente, a elevada proporção de violência familiar encontrada na clientela por meio de busca ativa não foi captada pelo fluxo "espontâneo" do ambulatório de pediatria. Os resultados obtidos por intermédio da aplicação de instrumentos de aferição específicos como as CTS revelaram uma elevada freqüência de casos, tanto na relação do casal, como deste para com a criança-índice. O uso de agressão psicológica contra a criança foi referido pela maioria das respondentes, também tendo sido elevada a prevalência de maus-tratos físicos graves na resolução dos conflitos existentes entre os casais. No entanto, estes achados não estão em sinto- nia com a casuística reportada pelo serviço social do ambulatório de pediatria. Mesmo considerando apenas os casos graves e supostamente mais evidentes, observa-se uma expressiva diferença entre os números do serviço social e os revelados pela busca ativa. A aplicação da CTSPC identificou uma prevalência de 10,0\% de agressão física grave contra a criança perpetrada pela mãe, o que corresponderia a uma projeção de aproximadamente 124 casos na população que procurou consulta de primeira vez no ambulatório pediátrico durante a janela temporal considerada na pesquisa. No entanto, somente 14 casos de maus-tratos físicos foram encaminhados ao serviço social no período, cerca de 11,0\% da provável prevalência.

Uma nítida divergência também pode ser observada entre a prevalência de agressão psicológica identificada ativamente e os raros casos encaminhados ao serviço social. Efetivamente, não se esperariam muitos eventos de agressão psicológica sendo referidos aos setores competentes, mas a discrepância entre as duas fontes de informação é marcante. Vale ressaltar que a maioria dos casos reportados pelo serviço social foi de abuso sexual, uma forma de violência contra a criança que não foi ativamente avaliada nesta pesquisa de clientela. Esta faceta importante da violência íntima mereceria mais atenção e esforços futuros.

Os resultados referentes à ocorrência de violência marital foram alarmantes, inclusive em relação a atitudes consideradas de extrema violência, como é o uso de armas de fogo. Este cenário identifica a exposição de crianças a ambientes de alto risco para o desenvolvimento de lesões, sejam estas intencionais ou não. Ademais, crianças que testemunham a violência entre seus pais ou cuidadores experimentam uma série de sensações negativas que po-
Tabela 1

Casos de violência contra a criança registrados pelo serviço social do ambulatório de pediatria do Hospital Universitário Pedro Ernesto, Rio de Janeiro, Brasil, no período de junho de 2000 a maio de 2001.

\begin{tabular}{lcc}
\hline Tipos de violência & Casos & Prevalência (\%)* \\
\hline Agressão física & 14 & $1,1(0,6-1,9)$ \\
Agressão psicológica & 3 & $0,2(0,0-0,7)$ \\
Abuso sexual & 22 & $1,8(1,1-2,7)$ \\
Negligência & 2 & $0,2(0,02-0,6)$ \\
Total & 41 & $3,3(2,4-4,5)$ \\
\hline
\end{tabular}

* Valores baseados em $n=1.241$ consultas realizadas no período.

Entre parênteses: intervalo de confiança de $95 \%$ obtido via teste exato de Fisher. 
dem resultar no aparecimento de baixo rendimento escolar, distúrbios de conduta, agressividade, baixa auto-estima, transtornos no sono e doenças somáticas crônicas, entre outros 36, 37,38 . O impacto negativo sobre a saúde da criança é ainda maior quando a violência íntima está presente em relações que envolvem indivíduos com fortes vínculos afetivos, como os pais ou figuras parentais 39 . A violência íntima contra a mulher, se já não deletéria para a própria, merece ser considerada como um agravo direto à saúde da criança, sendo a sua investigação uma importante ferramenta na detecção de maus-tratos nesta faixa etária 40,41 . Os resultados encontrados na pesquisa estão em sintonia com a literatura que aponta para a existência de co-ocorrência da violência familiar contra a criança e contra a mulher, justificando a incorporação do questionamento deste tipo de violência em serviços de assistência à criança $22,37,42$. Vale reparar que, a despeito da ocorrência de graves conflitos maritais, nenhum caso foi referido ao serviço social, indicando se tratar de algo que ainda passa completamente despercebido pelos profissionais atuantes.

Como visto, ao se confrontar as informações de busca ativa com a casuística espontânea do ambulatório de pediatria, observa-se que um número expressivo de situações de violência familiar contra a criança deixou de ser detectado pelos profissionais do setor. Presentemente, a violência de foro íntimo é dificilmente identificada por meio das abordagens rotineiramente empregadas em serviços de saúde, que tendem a uma perspectiva assistencialista baseada em práticas curativas fundamentadas preferencialmente na observação de sinais e sintomas de quadros clínicos. Como sugerem os resultados, casos graves e explosivos em sua apresentação podem eventualmente até ser captados e referidos. No entanto, o mesmo não ocorre nas situações menos evidentes que, potencialmente, podem vir a se agravar no futuro e cujo enfrentamento poderá se tornar mais trabalhoso para todas as partes envolvidas. São estas as oportunidades perdidas, os quadros latentes de conflito familiar, que deixam de ser identificados em consultas regulares da prática pediátrica e que, a rigor, envolvem prioritariamente problemas cotidianos no âmbito da prática pediátrica.

As razões para o baixo índice de identificação e encaminhamento de eventos podem ser atribuídas a diversas fontes. Uma destas se refere à formação e atuação de profissionais da área da saúde. A violência familiar demanda avaliações amplas, tanto no âmbito social quanto no psicológico. A apresentação dos casos de violência familiar que chegam aos serviços de saúde pode ser muito distinta, variando desde queixas vagas, envolvendo baixo rendimento escolar ou outras alterações comportamentais, até lesões corporais graves. Estas últimas são de fácil observação, já que correspondem ao padrão rotineiro na ótica assistencial. Já as outras formas de apresentação requerem um elevado grau de suspeição para sua adequada detecção, necessitando que o profissional esteja sensibilizado à questão. Muitas vezes ainda, evidências físicas de maus-tratos ou relatos típicos não são sequer visíveis, o que dificulta ainda mais este processo para profissionais de saúde que nem sempre estão capacitados para lidar com um evento desta natureza 29,43,44.

Um outro agravante seria a pouca ênfase nos cursos de graduação às questões relacionadas a temas que englobem aspectos para além do binômio saúde-doença. No lugar de agravos que, como visto, são comuns e graves como a violência familiar, o interesse maior se concentra em problemas mais "palpáveis" e/ou com soluções terapêuticas mais “objetivas”. Direta ou indiretamente, uma confluência de motivos de ordem pessoal também contribui neste processo. O pouco tempo que o profissional percebe dispor para o atendimento; o receio em ofender ao tocar no assunto; a percepção de que não existem recursos disponíveis para oferecer às vítimas soluções efetivas; acreditar que não faz parte de suas atribuições abordar o assunto; o sentimento de impotência diante do problema ou ainda, a sensação de estar abrindo uma "caixa de pandora" sem ter em vista uma seqüência de ações programadas são algumas das razões apontadas $25,28,40,41,45,46$. A violência familiar, ainda que reconhecidamente de difícil abordagem para o profissional da área da saúde, não deveria ser vista como um problema de menor importância se comparada a tantos outros agravos de menor magnitude, mas que, habitualmente, recebem atenção especial e são ativamente investigados 38,45 . As vítimas da violência esperam que seus cuidadores lhes ofereçam oportunidades para ultrapassar o muro do silêncio de forma segura, e caberia aos profissionais criar um ambiente propício a esta revelação. Neste sentido, o interesse em avaliar e conhecer as questões que envolvem a violência familiar e sua repercussão na saúde da criança requereria um estímulo ainda na etapa de formação e treinamento profissional 41, o que significa a inclusão de questões sociais e demográficas na grade curricular da formação na área da saúde.

Os resultados também suscitam uma reflexão sobre o papel dos serviços de saúde na 
abordagem da violência familiar. A atenção direcionada predominantemente aos casos graves e aberrantes, que inquestionavelmente necessitam de cuidado, é uma proposta incompleta, que acaba por excluir a possibilidade de uma visão mais ampla do fenômeno. As atividades desenvolvidas nos serviços de assistência primária estariam se limitando ao acompanhamento das vítimas e de suas famílias, na tentativa de controlar os desfechos já instalados decorrentes da violência. Sabe-se, no entanto, que este não é o único papel a ser desempenhado por estas instituições e que ações preventivas e educativas também merecem ser contempladas em suas diretrizes 18,19,41. Esta perspectiva mais abrangente de abordagem demanda mudanças, tanto nas estruturas e ambientes, quanto nas rotinas de trabalho empregadas em serviços de atendimento primário. Para o seu pleno desenvolvimento, faz-se necessário um redirecionamento do olhar dos profissionais envolvidos, que passariam a avaliar sua clientela dentro de um contexto que abarque também aspectos relacionados ao bem-estar de toda a família 47. A atuação "confortável" do setor saúde que enfatiza os agravos em que se "conhece claramente causas e efeitos", dá lugar ao desafio de abordar um fenômeno com implicações em terrenos desconhecidos.

Conforme sugerido na introdução deste artigo, apesar da presente avaliação se restringir a um único serviço, é possível supor que o cenário observado tenda ao encontrado em vários outros serviços de saúde de mesmo porte e tipo de adstrição. Vale lembrar que, mesmo sendo um hospital universitário, o serviço focalizado tem atribuições e atividades características de assistência primária, não muito distintas de outros ambulatórios de um centro de saúde da rede pública da cidade do Rio de Ja- neiro. Em contrapartida, existe a particularidade decorrente das atividades docentes e, por se tratar de uma instituição de ensino supostamente diferenciada, a generalização dos achados mereceria uma certa cautela e parcimônia. No entanto, o quadro encontrado pode ser considerado ainda mais crítico, precisamente porque não se observa a irradiação de conhecimento e práticas que, em princípio, seriam desejadas numa instituição de ensino. Numa perspectiva ainda mais ampla, valeria perguntar se o quadro não é ainda mais sombrio em unidades sem essa tal diferenciação. Seja como for, as informações e questões suscitadas neste estudo merecem ser exploradas e corroboradas em outros centros e localidades.

Para além das necessárias replicações deste estudo, caberiam aqui algumas sugestões para pesquisas futuras com vista à identificação dos entraves encontrados nas várias etapas da abordagem da violência familiar, talvez o mais controverso e desafiante evento a ser confrontado na assistência primária à saúde 45 . Objetivando a minimização de oportunidades perdidas, um aspecto central a ser enfrentado se refere aos processos de detecção de situações latentes ou instaladas de violência familiar contra a criança. Buscar o aprimoramento de rotinas de rastreamento, bem como a adaptação e aperfeiçoamento de instrumentos para executá-las, mereceriam destaque nesta agenda de investigação. Outros aspectos também são prioritários no enfrentamento desta questão, tais como a formação de equipes multidisciplinares, o escopo e conteúdo do treinamento e educação continuada oferecidos a estas equipes, ou ainda, a criação de redes de suporte para as vítimas no que se refere ao monitoramento de situações de recrudescimento ou acirramento de episódios violentos 19,41,48,49,50.

\section{Resumo}

A violência familiar é considerada uma questão prioritária em saúde pública, sendo o problema ainda mais marcante na infância. Informações referentes à morbidade deste agravo podem estar subdimensionadas devido a entraves na detecção de casos. O objetivo deste estudo é contrastar a magnitude da violência contra a criança, aferida ativamente em um ambulatório, com a casuística espontânea do serviço. Foram realizadas 245 entrevistas entre abril e junho de 2001, utilizando-se as Conflict Tactics Scales: Parent-Child Version (CTSPC) e a Revised Conflict Tactics Scales (CTS2) para aferir os eventos violentos. Os casos encaminhados ao Serviço Social representaram a casuísti- ca do serviço no período da busca ativa (12 meses). Encontrou-se uma elevada prevalência de violência física entre o casal, com eventos graves ocorrendo em 17,0\% das famílias. Em relação à criança, agressões físicas "menores" foram referidas em 46,0\% de famílias, porquanto a forma grave, em 9,9\%. A prevalência identificada espontaneamente foi de 3,3\%. Este estudo de caso mostra as oportunidades perdidas de detecção e chama-se a atenção para a necessidade de rever a abordagem da violência familiar em serviços de saúde.

Criança; Violência Doméstica; Serviços de Saúde 


\section{Colaboradores}

A. T. M. S. Moura participou da elaboração do projeto, supervisão do trabalho de campo, análise dos dados e redação do artigo. M. E. Reichenheim contribuiu no planejamento do projeto, análise dos dados e redação do artigo.

\section{Referências}

1. Lima MLC, Ximenes R. Violência e morte: diferenciais da mortalidade por causas externas no espaço urbano do Recife, 1991. Cad Saúde Pública 1998; 14:829-40.

2. Barros MD, Ximenes R, Lima ML. Mortalidade por causas externas em crianças e adolescentes: tendências de 1979 a 1995. Rev Saúde Pública 2001; 35:142-9.

3. Costa MCN, Mota ELA, Paim JS, Silva LMV, Teixeira MG, Mendes CMC. Mortalidade infantil no Brasil em períodos recentes de crise econômica. Rev Saúde Pública 2003; 37:699-706.

4. Mello-Jorge MHP, Gawryzewski VP, Latorre MR. Análise de dados de mortalidade. Rev Saúde Pública 1997; 31:5-24

5. Secretaria de Saúde do Estado do Rio de Janeiro. Indicadores e informações em saúde. Rio de Janeiro: Secretaria de Saúde do Estado do Rio de Janeiro; 2000.

6. Reichenheim ME, Werneck GL. Anos potenciais de vida perdidos no Rio de Janeiro, 1990. As mortes violentas em questão. Cad Saúde Pública 1994; 10 Suppl 1:188-98

7. Sociedade Brasileira de Pediatria. Guia de atuação frente a maus-tratos na infância e na adolescência. Rio de Janeiro: Sociedade Brasileira de Pediatria/Centro Latino-Americano de Estudos de Violência e Saúde Jorge Carelli, Escola Nacional de Saúde Pública, Fundação Oswaldo Cruz; 2001.

8. Straus MA, Gelles RJ. Physical violence in american families: risk factors and adaptations to violence in 8145 families. New Brunswick: Transaction Publisher; 1995.

9. Barnett OW, Miller-Perrin CL, Perrin R. Family violence across the lifespan: an introduction. London: Sage Publications; 1997.

10. Noel NL, Yam M. Domestic violence. The pregnant battered women. Nurs Clin North Am 1992; 27:871-84

11. Deslandes SF. Atenção a crianças e adolescentes vítimas de violência doméstica: análise de um serviço. Cad Saúde Pública 1994; 10 Suppl 1:177-87.

12. Assis SG. Crianças e adolescentes violentados: passado, presente e perspectivas para o futuro. Cad Saúde Pública 1994; 10 Suppl 1:126-34.

\section{Agradecimentos}

O projeto de pesquisa no qual se insere este estudo teve o apoio parcial do Conselho Nacional de Desenvolvimento Científico e Tecnológico, Projeto PRONEX, auxílio n. 663073/9987. M. E. R. tem apoio do CNPq, auxílio n. 300234/94-5. Os autores agradecem aos entrevistadores Mariana Silva Oliveira e Vitor de Mello Netto pela valiosa contribuição na fase de coleta de dados.

13. Moraes CL, Reichenheim ME. Domestic violence during pregnancy in Rio de Janeiro, Brazil. Int J Gynaecol Obstet 2002; 79:269-77.

14. Monteiro Filho L, Phebo LB. Maus-tratos contra crianças e adolescentes. Proteção e prevenção. Guia para profissionais de saúde. Rio de Janeiro: Associação Brasileira de Proteção à Infância e Adolescência; 1992.

15. Krug EG, Dahlberg LL, Mercy JA, Zwi AB, Lozano R. World report on violence and health. Geneva: World Health Organization; 2002.

16. Secretaria de Políticas de Saúde, Ministério da Saúde. Violência intrafamiliar: orientações para prática em serviço. Brasília: Ministério da Saúde; 2002. (Cadernos de Atenção Básica 8).

17. Boyle A, Robinson S, Atkinson P. Domestic violence in emergency medicine patients. Emerg Med J 2004; 21:9-13.

18. World Health Organization. WHO recognizes child abuse as a major public health problem. Geneva: World Health Organization; 1999.

19. Djeddah C, Facchin P, Ranzato C, Romer C. Child abuse: current problems and key public health challenges. Soc Sci Med 2000; 51:905-15.

20. Reichenheim ME, Hasselmann MH, Moraes CL. Conseqüências da violência familiar na saúde da criança e do adolescente: contribuições para a elaboração de propostas de ação. Ciênc Saúde Coletiva 1999; 4:109-21.

21. Wright JL, Cheng TL. Successful approaches to community violence intervention and prevention. Pediatr Clin North Am 1998; 45:459-67.

22. Heyman RE, Slep AM. Risk factors for family violence: introduction to the special series. Aggress Violent Behav 2001; 6:115-9.

23. Black DA, Slep AMS, Heyman RE. Risk factors for child psychological abuse. Aggress Violent Behav 2001; 6:189-201.

24. Petridou E, Browne A, Lichter E, Dedoukou X, Alexe D, Dessypris N. What distinguishes unintentional injuries from injuries due to intimate partner violence: a study in Greek ambulatory care settings. Inj Prev 2002; 8:197-201.

25. Olson L, Anctil C, Fullerton L, Brillman J, Arbuckle J, Sklar D. Increasing emergency physician recog- 
nition of domestic violence. Ann Emerg Med 1996; 27:741-6.

26. Benger JR, Pearce V. Simple intervention to improve detection of child abuse in emergency departments. BMJ 2002; 324:780.

27. Wiist WH, McFarlane J. The effectiveness of an abuse assessment protocol in public health prenatal clinics. Am J Public Health 1999; 89:1217-21.

28. Erickson MJ, Hill TD, Siegel RM. Barriers to domestic violence screening in the pediatric setting. Pediatrics 2001; 108:98-102.

29. Thompson RS, Rivara FP, Thompson DC, Barlow WE, Sugg NK, Maiuro RD, et al. Identification and management of domestic violence: a randomized trial. Am J Prev Med 2000; 19:253-63.

30. Straus MA, Hamby SH, Warren WL. The Conflict Tactics Scales handbook. Los Angeles: Western Psychological Services; 2003.

31. Straus MA, Hamby SL, Boney-McCoy S, Sugarman D. B. The Revised Conflict Tactics Scales (CTS2). Development and preliminary psychometric data. J Fam Issues 1996; 17:283-316.

32. Reichenheim ME, Moraes CL, Hasselmann MH Semantic equivalence of the portuguese version of the abuse assessment screen tool used for the screening of violence against pregnant women. Rev Saúde Pública 2000; 34:610-6.

33. Reichenheim ME, Moraes CL. Adaptação transcultural do instrumento Parent-Child Conflict Tactics Scales (CTSPC), utilizado para identificar a violência contra a criança. Cad Saúde Pública 2003; 19:1701-12.

34. Moraes CL, Hasselmann MH, Reichenheim ME. Adaptação transcultural para o português do instrumento "Revised Conflict Tactics Scales (CTS2)" utilizado para identificar a violência entre casais. Cad Saúde Pública 2002; 18:163-76.

35. Shrout PE. Measurement reliability and agreement in psychiatry. Stat Methods Med Res 1998; 7:301-17.

36. Mace SE, Gerardi MJ, Dietrich AM, Knazik SR Mulligan-Smith D, Sweeney RL, et al. Injury prevention and control in children. Ann Emerg Med 2001; 38:405-14.

37. Kerker BD, Horwitz SM, Leventhal JM, Plichta S, Leaf PJ. Identification of violence in the home: pediatric and parental reports. Arch Pediatr Adolesc Med 2000; 154:457-62.
38. Eisenstat SA, Bancroft L. Domestic violence. N Engl J Med 1999; 341:886-92.

39. Knapp JF. The impact of children witnessing violence. Pediatr Clin North Am 1998; 45:355-64.

40. Dowd MD, Kennedy C, Knapp JF, StallbaumerRouyer J. Mothers' and health care providers' perspectives on screening for intimate partner violence in a pediatric emergency department. Arch Pediatr Adolesc Med 2002; 156:794-9.

41. Borowsky IW, Ireland M. Parental screening for intimate partner violence by pediatricians and family physicians. Pediatrics 2002; 110:509-16.

42. Slep AMS, Heyman RE. Where do we go from here? Moving toward an integrated approach to family violence. Aggress Violent Behav 2001; 6:353-6.

43. Adams JA, Harper K, Knudson S, Revilla J. Examination findings in legally confirmed child sexual abuse: it's normal to be normal. Pediatrics 1994; 94:310-7.

44. Freitag R, Lazoritz S, Kini N. Psychosocial aspects of child abuse for primary care pediatricians. Pediatr Clin North Am 1998; 45:391-402.

45. Gerbert B, Gansky SA, Tang JW, McPhee SJ, Carlton R, Herzig K, et al. Domestic violence compared to other health risks: a survey of physicians' beliefs and behaviors. Am J Prev Med 2002; 23:82-90.

46. Gough D. Defining the problem. Child Abuse Negl 1996; 20:993-1002.

47. Dubowitz H. Preventing child neglect and physical abuse: a role for pediatricians. Pediatr Rev 2002; 23:191-6.

48. Calhoun AD, Clark-Jones F. Theoretical frameworks. Developmental psychopathology, the public health approach to violence, and the cycle of violence. Pediatr Clin North Am 1998; 45:281-91.

49. Glass N, Dearwater S, Campbell J. Intimate partner violence screening and intervention: data from eleven Pennsylvania and California community hospital emergency departments. J Emerg Nurs 2001; 27:141-9.

50. Onyskiw JE, Harrison MJ, Spady D, McConnan L. Formative evaluation of a collaborative community-based child abuse prevention project. Child Abuse Negl 1999; 23:1069-81.

Recebido em 18/Jun/2004

Versão final reapresentada em 26/Jan/2005

Aprovado em 03/Fev/2005 